\title{
"B̆ASCENDER
}

Trascender, Contabilidad y Gestión Núm. 9 (septiembre - diciembre del 2018). ISSN: 2448-6388. Universidad de Sonora. Departamento de Contabilidad. Reserva de Derechos 04-2015-04172070800-203.

\section{Ética en la Administración Pública: Valores, Transparencia e Integridad de los Servidores Públicos en el Estado de Sonora}

Ethics in public administration: values, transparency and integrity of public servants in the state of Sonora

$$
\text { Vladimir Atondo De la Vara }{ }^{1}
$$

\section{Resumen}

El presente ensayo de investigación trata de cómo el descuido de la ética en el actuar de los servidores públicos ha permitido el aumento de los actos de corrupción dentro de la administración pública en el Estado de Sonora; se enfoca además en como la corrupción afecta la eficiencia de las instituciones públicas y como se ha visto afectada la imagen del desempeño del gobierno y de los servidores públicos. Es una investigación de tipo descriptiva y correlacional ya que aborda diferentes enfoques como la normatividad existente donde se definen cuáles son las faltas administrativas graves en que incurren los empleados de la administración pública, así como las sanciones estipuladas para dichas fallas.

La metodología utilizada en la realización de este proyecto corresponde al tipo documental, ya que se tomó como base investigaciones, artículos y tesis de diferentes bases de datos especializadas. Muestra como en los dos últimos años el número de demandas presentadas se ha mantenido prácticamente sin cambios con un promedio de 224 casos de actos de investigación administrativa, sin embargo de 2009 a la fecha se han concretado 1,090 sanciones por más de 6 mil 440 millones de pesos.

Por último trata de la importancia de la formación de valores éticos y el establecimiento de un perfil público para el reclutamientos de trabajadores de gobierno; que junto con el robustecimiento de las reformas legales como es el Sistema Anticorrupción Sonora, la declaración patrimonial tres de tres, la transparencia y rendición de cuentas ayudan a generar confianza en la ciudadanía.

Palabras clave: ética, servidores públicos, administración pública, corrupción.

DOI: https://doi.org/10.36791/tcg.v0i9.39

Recibido: 14 de abril de 2018.

Aceptado: 16 de mayo de 2018.

\footnotetext{
${ }^{1}$ Vladimir Atondo De la Vara. Contador Público. Auditor Supervisor del Instituto Superior de Auditoria y Fiscalización (ISAF). Correo: vladimir_atondo@hotmail.com
} 


\section{Abstract}

The present research essay deals with how the neglect of ethics in the actions of public servants has allowed the increase of acts of corruption within the public administration in the State of Sonora; it also focuses on how corruption affects the efficiency of public institutions and how the image of the performance of government and public servants has been affected. It is a descriptive and correlational type of research since it approaches different points of view such as the existing regulations in which are defined the serious administrative faults incurred by public administration employees, as well as the sanctions stipulated for said faults.

The methodology used in the realization of this project corresponds to the documentary type, since it was based on research, articles and theses of different specialized databases. It shows how in the last two years the number of demands presented has remained practically unchanged with an average of 224 cases of administrative investigation acts, however from 2009 to date there have been 1,090 sanctions for more than 6 thousand 440 million pesos.

Finally, it deals with the importance of the formation of ethical values and the establishment of a public profile for the recruitment of government workers; that together with the strengthening of legal reforms such as the Sonora Anti-Corruption System, the patrimonial declaration three of three, transparency and accountability help to generate trust in the citizenry.

Keywords: ethics, public servants, public administration, corruption. 


\section{Introducción}

De acuerdo con Martiñez Navarro (2011: 85):

"En los últimos años, los países de América Latina han experimentado reformas importantes en el Estado y particularmente en la Administración Pública. En este sentido, la cuestión de la ética aparece junto a estas transformaciones y cambios. La adopción de códigos, de regulaciones éticas, así como de sistemas de transparencia y rendición de cuentas es prueba del rol que ha venido jugando la ética en todos estos procesos".

En México, los temas relacionados con el combarte a la corrupción, la transparencia y rendición de cuentas han cobrado gran importancia en el debate público, esto debido a que la corrupción es un mal costoso para la sociedad ya que merma el desarrollo económico, al disminuir la inversión, la calidad de los servicios prestados y la infraestructura gubernamental, lo que a su vez afecta la calidad de vida de la sociedad (Atondo, 2018); este trabajo trata sobre la importancia de la actuación de los servidores públicos al poner en práctica su ética pública.

\section{Antecedentes}

La globalización que se ha vivido en los últimos años, ha traído consigo el incremento de las demandas sociales, en México se han presentado situaciones que han cambiado a la sociedad, económica, política y culturalmente, entre los que podemos mencionar actos de corrupción que involucran en su mayoría a políticos y titulares o directores de organismos que ha quedado en la impunidad, lo que ha provocado el hartazgo de la ciudadanía que no cree en el gobierno ni en sus representantes, lo que ha ocasionado el establecimiento de numerosos cambios legislativos como la adopción del Sistema Estatal Anticorrupción y el reforzamiento en cuanto a la obligación de presentar periódicamente el resultado del ejercicio y aplicación de recursos conforme a la Ley de Transparencia y Acceso a la Información Pública del Estado de Sonora.

Los reclamos sociales que ha traído la globalización ejercen cada día más presión, pues representan las áreas de oportunidad para la administración pública, por lo que el Estado se ha visto en la necesidad de adoptar programas y estrategias acordes con la realidad, así como reforzar principios éticos y valores en los servidores públicos durante su actuar para lograr un mejor imagen y desempeño institucional.

La ética es la parte de la filosofía que estudia la moralidad de los actos humanos, según los expuesto por Diego Bautista (2007, Pag 22) es la disciplina del conocimiento que estudia las actitudes, hábitos y costumbres del ser humano. Estudia y explica el porqué de la conducta humana y propone formas deseables para actuar. "La ética es la morada o lugar donde se habita, es una lámpara que ilumina al hombre en su vida, la raíz donde se sujeta el individuo frente a los vientos de las pasiones. Se posee ética cuando se logra establecer, para uno mismo, un conjunto de virtudes que se tornan principios bajo los cuales se rige la conducta. El ethos del hombre es la tierra fértil de donde brotan todos los actos humanos que dan fundamento a una forma o modo de vida.

Dentro de la administración pública, el objetivo de la ética es la adopción de códigos de valores y conducta tomando como base la integridad, la honradez, el honor y la cortesía de los servidores públicos para robustecer las estructuras institucionales.

\section{Problema de Investigación}

En el manejo y ejecución de los recursos públicos se presentan conductas de incumplimiento por parte de los funcionarios públicos; por lo que es necesaria la profesionalización y capacitación basada en ética y valores para Servidores Públicos de la 
Administración Pública en Estado de Sonora como herramienta para coadyuvar a mitigar la corrupción. Analizado desde 2009.

\section{Justificación}

El estudio de la ética resulta importante porque aporta valores que ayudan a mejorar la conducta social y el desempeño institucional, analizar el actuar de los servidores públicos supone un acercamiento entre la administración pública y la ciudadanía con el fin de lograr un mejor entendimiento entre ambas partes, y del cual se deriven beneficios mutuos.

Para la gestión pública: formular programas apegados a las necesidades sociales existentes, conocer el entono social para mejorar la toma de decisiones, fortalecer los mecanismos de transparencia y rendición de cuentas.

Para la sociedad: reforzar el ejercicio ciudadano, realizar aportaciones o establecer demandas sociales con el objeto de provocar cambios culturales que se reflejen en la definición de nuevas políticas públicas.

Según la Secretaría de la Contraloría General, (2014) es necesaria la implementación de un código de Conducta que permita alcanzar la excelencia en el comportamiento humano y laboral se requiere promover y desarrollar una cultura basada en valores éticos y morales que ayuden al cumplimiento correcto de las funciones y deberes propios del personal. Se busca propiciar que el servicio público se realice en un alto nivel que incluya la mejora continua en el actuar personal, desempeño con eficiencia, eficacia y transparencia, así como mantener un ambiente de cordialidad en el desempeño laboral.

Dentro de la administración pública resulta evidente la falta de un departamento o área que promueva y enaltezca los valores éticos que deben normar el actuar de los funcionarios públicos durante la realización de su trabajo, la adopción de valores trae consigo el desarrollo conductas apropiadas para quienes prestan sus servicios a la comunidad y los grupos de interés con los que se relacionan.

\section{Objetivo}

Realizar un diagnóstico de las conductas presentes en los empleados de la administración pública que fomentan la corrupción en el Estado de Sonora y como la formación de valores éticos y profesionalización ayuda a recuperar la confianza de la ciudadanía.

\section{Limitación}

La limitación principal encontrada en el desarrollo de esta investigación es la falta de detalle en la información correspondiente a las sanciones interpuestas a los servidores públicos por parte de la Secretaría de la Contraloría General del Estado de Sonora, si bien, en el Anexo Estadístico Grafico del Segundo Informe de Gobierno de fecha septiembre 2017, se informa que de septiembre de 2009 a septiembre de 2017 se han interpuesto 1,090 sanciones, pero, no se especifica cuáles son las faltas administrativas graves que las originaron.

\section{Metodología}

El método utilizado para la realización de este trabajo, consistió en la obtención de información sobre el tema de estudio de diversas fuentes como libros, revistas, artículos de conferencias, bases de datos especializadas en trabajos de investigación como Ebsco, Thomson Reuters, Dialnet, Springer, Google Académico entre otros.

El enfoque deductivo utilizado en la investigación sirvió para clasificar la literatura por su país de origen, de manera cronológica, acorde al tema de interés, hasta delimitar y definir de manera clara el problema de investigación. 


\section{Análisis de la Información}

\section{Acercamiento al concepto de Ética}

Son varios los filósofos que mostraron interés en la ética, Aristóteles centra sus estudios en que el hombre por vivir en comunidad debe desarrollar el control de sus instintos por medio de principios y valores, por su parte Confucio creía que el hombre debe mostrar una profunda moralidad y conocimiento profundo de sus deberes tratándose de servidores públicos.

Podemos decir que el equilibrio ético se da cuando una persona es capaz de combinar la razón y la pasión, al hacer esto, se logra un autodesarrollo ordenado que lo lleva a la virtud.

El fundamento principal en la ética es la autonomía, es el asumir normas válidas para uno mismo y para los demás para conseguir un bien para la sociedad, la moral nos ayuda a establecer el tipo de vida y acciones que debemos escoger.

En el caso de los servidores públicos, es común que sus puntos de vista e interpretación de las diferentes situaciones en su actuar cambie o evolucione conforme su madurez, experiencia, formación profesional, entorno socioeconómico, valores, familia, etc. le sirven para formar y explicar su propio enfoque de las cosas.

\section{Objeto de la Ética}

La ética pública estudia y analiza el perfil, la formación y el comportamiento responsable $\mathrm{y}$ comprometido de las personas que se ocupan de los asuntos públicos, generando un cambio de actitud en ella al inculcarles valores de servicio público. Según (Naessens, 2010).

\section{Formación de los Servidores Públicos}

Otro aspecto a considerar es la formación o profesionalización de los servidores públicos, por lo que surge la pregunta, ¿qué grupo de empleados públicos son los que deben recibir formación ética?, la respuesta es fácil, todos los empleados; esta no es un tarea nada fácil, por el contrario, realizarla implica el consumo de recursos que no están presupuestados y además consume gran cantidad de tiempo, actualmente algunos países han adoptado medidas para la formación de valores y establecen grupos de capacitación para nuevos servidores públicos, empleados en áreas de riesgo, y servidores con altas jerarquías como titulares y jefe de departamento.

Con base a lo anterior expuesto, resulta imprescindible que sean los servidores públicos los que deben adoptar los principios éticos y asegurar su aplicación en su actuación profesional, en su relación con los ciudadanos y con la administración pública en general.

\section{El papel de la Administración Pública}

La Administración pública presta servicio a la sociedad y es ésta su verdadera razón de ser, es decir, atender los intereses y derechos de los ciudadanos y los diferentes grupos que la componen, buscando asimismo el equilibrio de todos ellos, compatible con los principios de legalidad, eficacia y eficiencia. Como consecuencia, la organización debe orientar sus procesos a satisfacer las necesidades y expectativas de los ciudadanos. (Seijo Suárez \& Añez Tellería, 2008).

La función pública debe estar enfocada principalmente a ofrecer servicios de calidad a la ciudadanía, y debe diseñar los procesos en busca de la optimización de su función. La administración y los poderes públicos deben en todo momento unir fuerzas para juntos lograr un beneficio social.

En este orden de ideas, lo que se requiere es encaminar las acciones y metas existentes de los organismos y dependencias para implementar planes que sean medibles y cuantificables, que sus programas estén enfocados a cumplir realmente con la misión con la que fueron creados, la cual dicho sea de paso, debe ser brindar una visión clara para el servidor público se enfoque en el "deber ser" y ofrecer abiertamente el resultado de su desempeño y 
aplicación de recursos con el fin de recuperar la confianza de la ciudadanía, es decir, encontrar nuevos medios de valorar la integridad en el actuar de los servidores públicos por parte los ciudadanos y que su interacción sea apegada a la legalidad, lo cual brindará mejoras en la Administración pública.

Otro aspecto importante que se debe considerar para mejorar la administración pública es al momento de realizar el proceso de contratación de nuevos aspirantes, se debe establecer un perfil público deseable a fin de garantizar el buen desempeño del servidor. Es por eso que mientras se carezca de una forma eficiente de reclutar aspirantes al servicio público con autentica integridad y que antepongan el interés general al propio, la responsabilidad seguirá en los hombros funcionarios públicos o directivos encargados de la operación de las instituciones, responsabilidad que incluye la revisión y supervisión de sus subordinados a fin de evitar incompetencias en su actuar y posibles actos de corrupción.

\section{La corrupción: el desvío de la Ética}

El olvido de la ética en el ámbito público ha acarreado innumerables problemas siendo el más importante la corrupción, no solo en nuestro estado, sino en todo México y pudiera considerarse como un fenómeno de clase mundial; es un vicio que se ha implantado en la mayoría de las sociedades modernas y cuya característica es el desvío de recursos dentro de los gobiernos.

Acorde con Secretaria de la Función Pública (2013), la corrupción Consiste en el abuso del poder para beneficio propio. Puede clasificarse en corrupción a gran escala, menor y política, según la cantidad de fondos perdidos y el sector en el que se produzca.
La corrupción a gran escala consiste en actos cometidos en los niveles más altos del gobierno que involucran la distorsión de políticas o de funciones centrales del Estado, y que permiten a los líderes beneficiarse a expensas del bien común; Los actos de corrupción menores consisten en el abuso cotidiano de poder por funcionarios públicos de bajo y mediano rango al interactuar con ciudadanos comunes, quienes a menudo intentan acceder a bienes y servicios básicos en ámbitos como hospitales, escuelas, departamentos de policía y otros organismos; mientras que la Corrupción Política consiste en la Manipulación de políticas, instituciones y normas de procedimiento en la asignación de recursos y financiamiento por parte de los responsables de las decisiones políticas, quienes se abusan de su posición para conservar su poder, estatus y patrimonio.

"La corrupción por tanto, tiene lugar frecuentemente en las sociedades donde los empleados públicos tienen un considerable poder discrecional, rinden cuentas de forma limitada y hay poca transparencia en las operaciones del gobierno; en tales sociedades, las instituciones de la sociedad civil y el sector privado independientemente a menudo son débiles o están poco desarrolladas.” (Bautista, 2005: 54).

Adicionalmente de acuerdo al Índice de Percepción de la Corrupción 2017, México se encuentra entre los 5 países con mayor percepción de corrupción en América Latina, superado únicamente por Venezuela, Haití, Nicaragua y Guatemala, a nivel mundial se localiza en el número 135 de 180 países incluidos en el estudio. (Ver Figura 1, Percepción sobre niveles de corrupción). 
Figura 1. Percepción sobre niveles de corrupción

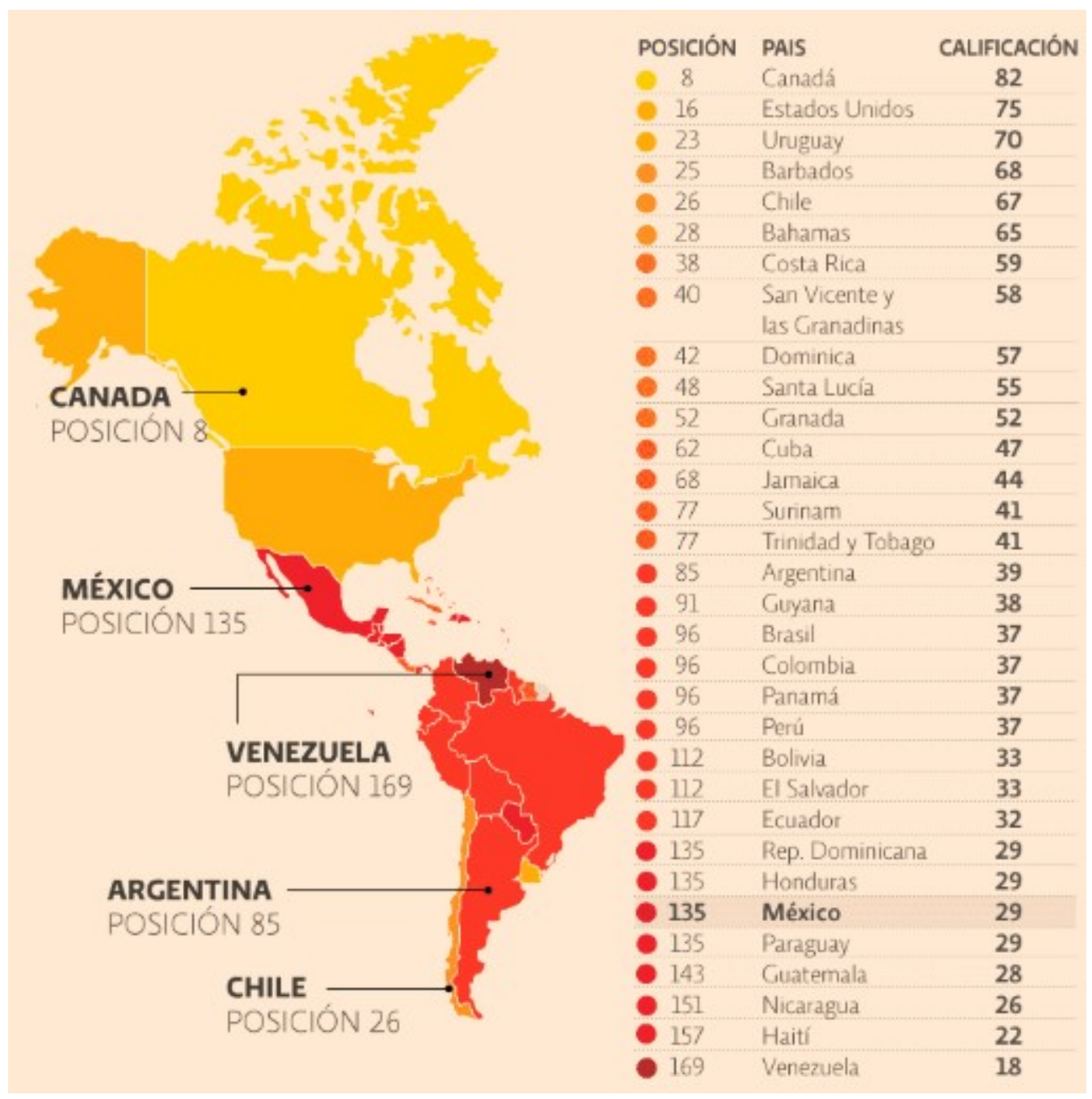

Fuente: Periódico el Economista, 2018.

En Sonora, México, se han presentado en los últimos años importantes actos de corrupción que involucran en su mayoría a políticos gobernantes y titulares o directores de organismos centralizados y descentralizado que ha quedado en la impunidad, entre los que podemos mencionar el endeudamiento de municipios, desvío de recursos, enriquecimiento ilícito, obras públicas realizadas con menores niveles de calidad, etc. esto, ha provocado el hartazgo de la ciudadanía que no cree en el gobierno ni en sus representantes; parte de las reformas adoptadas por nuestro país y que hoy en día cobran gran importancia resulta ser la transparencia y la rendición de cuentas, que consiste en mostrar de manera periódica el resultado de la administración de los recursos, regularmente en un portal de internet al que tiene acceso toda la población, para reforzar esta obligación se creó el Instituto Nacional de Transparencia, Acceso a la Información y Protección de Datos Personales (INAI), que representa el 
vínculo por el cual una persona puede solicitar información a cualquier entidad de gobierno que tiene la obligación de proporcionarla en el plazo establecido.

Entre las principales fuentes que originan la Corrupción podemos mencionar:

- Falta de perfil adecuado para ocupar un puesto de Servidor Público

- Inestabilidad laboral

- Sentido de pertenencia de los recursos públicos

- Baja remuneración

- Existencia de un sistema de rendición de cuentas adecuado

- Impunidad

\section{Delitos Graves de los Servidores Públicos en el} Estado de Sonora

De acuerdo con la Ley Estatal de Responsabilidades (Congreso del Estado Libre y Soberano de Sonora,
2017), en su Título IV De las faltas administrativas de los servidores públicos y actos de particulares vinculados con faltas administrativas graves, Capítulo II De las faltas administrativas graves de los Servidores públicos, en los artículos 91 al 104, establece como delitos los siguientes: cohecho, peculado, desvío de recursos, utilización indebida de información, abuso de funciones, conflicto de interés, contratación indebida, enriquecimiento ilícito, tráfico de influencias, encubrimiento, desacato, por mencionar algunas.

De acuerdo con al Anexo Estadístico Grafico del Segundo Informe de Gobierno de la Gobernadora del Estado de Sonora correspondiente al período de septiembre de 2016 a septiembre de 2017, se interpusieron un total de 221 denuncias contra servidores públicos, seis menos que en el periodo anterior, a continuación se anexa tabla con el detalle de las instancias ante las que fueron interpuestas dichos actos de presunta responsabilidad administrativa. (Ver Tabla 1, Actos de investigación administrativa 2017).

Tabla 1. Actos de investigación administrativa 2017

septiembre 2016 - septiembre 2017

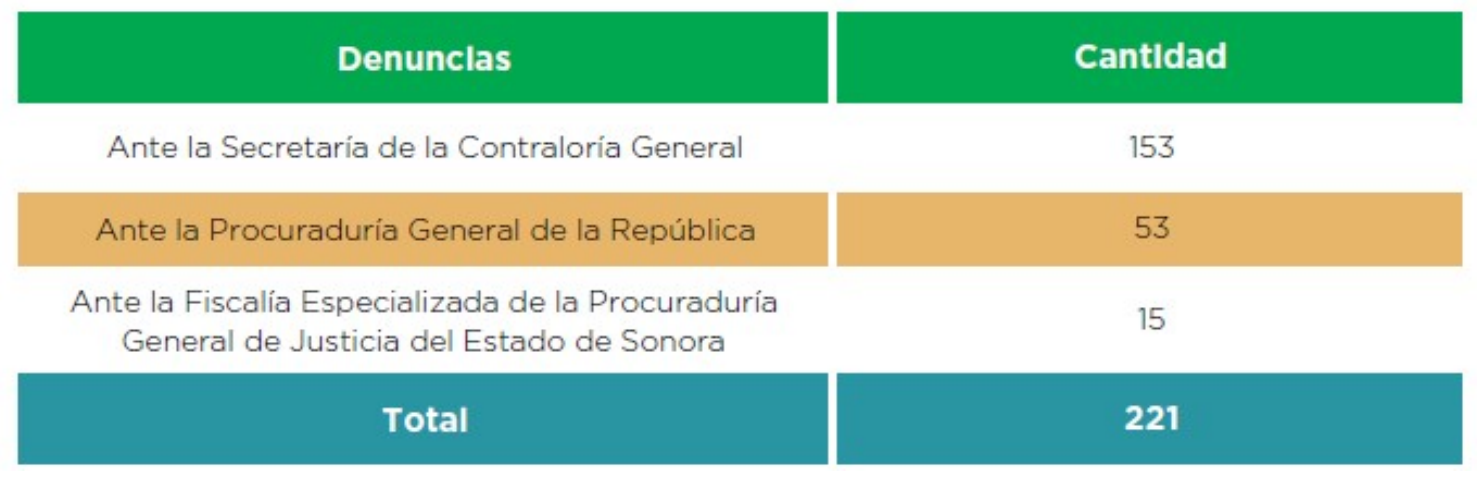

Fuente: Anexo estadístico gráfico del Segundo Informe de Gobierno 
Así mismo, en la siguiente tabla se detalla las sanciones impuestas por la Secretaría de la Contraloría General durante los dos últimos períodos de Gobierno Estatal, sumando 1,090 sanciones por 6 mil 440 millones 599 mil 300 pesos. Sin incluir 724 extrañamientos a Servidores Públicos por faltas administrativas no graves. (Ver tabla 2, Sanciones Emitidas por la Secretaría de la Contraloría General de 2009 a 2017).

Tabla 2. Sanciones emitidas por la Secretaría de la Contraloría General

$2009-2017$

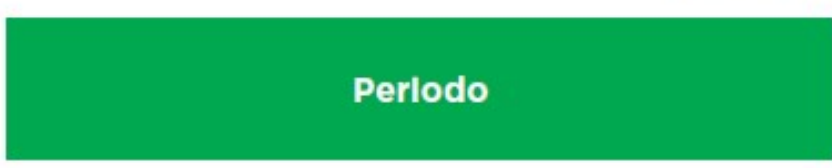

Septiembre 2009 a septiembre 2015

octubre 2015 a septiembre 2016

Octubre 2016 a septiembre 2017

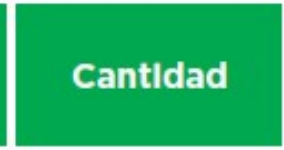

220
Monto de sanclón

económica

(miles de pesos)

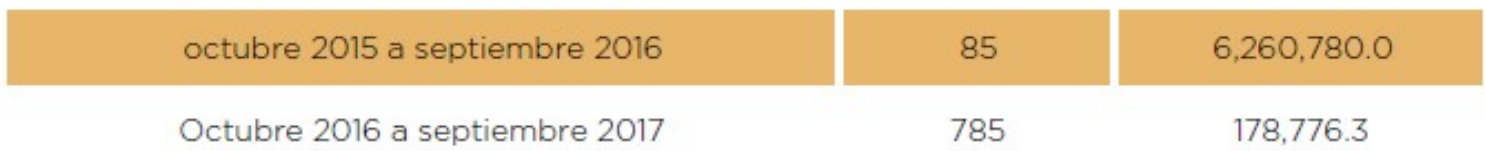

Fuente: Anexo estadístico gráfico del Segundo Informe de Gobierno

\section{El valor de la ética para generar confianza en la Administración Pública}

Restaurar y generar confianza entre los ciudadanos y sus instituciones ha de ser el objetivo fundamental y básico de la ética pública y para ello tiene que ser capaz de generar hábitos, convicciones y cambio de cultura. (Ausín Díez, 2015)

De acuerdo con Boehm (2015), la confianza no puede ser impuesta, se gana. Comunicar estándares éticos no es suficiente para generar confianza, se debe observar su cumplimiento en la conducta de los funcionarios públicos. ¿Pero cómo fomentar la conducta ética? Un código de comportamiento permite clarificar al funcionario, pero también a los ciudadanos, los estándares éticos y de conducta que deben guiar sus decisiones y acciones.

Los servidores públicos realizan actividades de cooperación y representan a las instituciones para las que laboran por lo que representan una gran influencia en la percepción de los ciudadanos, tienen que cumplir con las legislaciones existentes en su rol diario, por ello, el cambio de cultura organizacional debe ser uno de los objetivos principales de toda intervención ética dentro de la administración pública.

Confiar nos hace la vida más fácil, de manera que para fortalecer el servicio público se debe reforzar la ética de sus servidores ya que no es posible mantener una sociedad compleja sin la existencia de la confianza, son pasos difíciles que de principio parecerán imperceptibles, pero con voluntad y actitud puede lograrse para que en el largo plazo contemos con lo que actualmente se conoce como "buen gobierno".

La idea de buen gobierno está estrechamente unida en la actualidad a un sistema institucional que procura el pleno desarrollo de las personas y entidades, que garantiza un crecimiento económico sostenible y, en fin, que actúa en el ámbito propio de sus poderes mediante una actuaciones públicas ejemplares y en 
todo caso sometidas al control público y a la rendición de cuentas ante la ciudadanía. (Jimenez Asensio, 2013).

En otras palabras, es una apuesta hecha por la calidad de instituciones públicas que enfocan sus acciones en el servicio y por ende brinda bienestar a los ciudadanos al ver atendidas sus necesidades; la fortaleza en las instituciones representa una gran inversión pero debe forma parte de los planes estratégicos a largo plazo de los funcionarios encargados de la dirección de las diferentes entidades que conforman el Estado.

Acorde con Uvalle (2014) "La recuperación de la ética es asunto relevante, porque nutre y aporta el mejor desempeño de la gestión institucional, ya que con su contenido normativo y prescriptivo, contribuye a eliminar situaciones relacionadas con la opacidad, la corrupción, los desfalcos, la cleptocracia, el patrimonialismo y la ilegalidad”.

Hablar de ética del servicio público o del trabajo de los servidores públicos desde una perspectiva ética, no es hoy solamente una moda sino que supone hacer referencia a uno de los pilares de la reforma administrativa que está en marcha en todo el mundo. (Rodríguez-Arana Muñoz, 1997).

\section{Transparencia y Rendición de Cuentas}

En tiempos recientes, las demandas sociales se incrementan cada día, en parte por el acceso a la información que tienen los ciudadanos, así como por el auge de las redes sociales que son el medio por el cual mucha información (no necesariamente cierta), sale a la luz y forma parte del conocimiento informal, por medio del cual existen innumerables demandas hacia la clase política y las instituciones del Estado, esto ha orillado a los gobiernos a establecer $\mathrm{y}$ reformar reglas, procedimientos, leyes e incluso imponer y reforzar las sanciones para las conductas que se encuentran al margen de la ley.
"La transparencia la podemos considerar como la actividad que realizan los entes públicos por medio de la cual se enseña información a la sociedad con el objeto de que se mantenga informado y pueda utilizar lícitamente la información publicada o informada a través de los medios respectivos en forma impresa o electrónica”. (Sanromán, 2014: 9)

La lucha contra la corrupción en México, constituye uno de los pilares más fuertes para el desarrollo, es por eso que con la aprobación de la ley anticorrupción federal se han dado pasos para la réplica de dichas reformas a nivel estatal, Sonora es uno de los primeros estados hacer lo propio.

Los administradores públicos deben pensar en cómo dar coherencia y continuidad a las acciones que emprenden, para actuar con mayor capacidad estratégica, evitar desperdiciar recursos y obtener mejores resultados. Uno de los retos de futuro en este ámbito consiste en generar indicadores de ética pública ligados a instrumentos de gestión de la integridad, y de promoción y fiscalización de los mismos como base para orientar la actividad de funcionarios, particularmente en la fase de evaluación de los servicios públicos en el ámbito local. (Aldeguer, 2011)

Otra idea para robustecer la estructura actual del gobierno, es la implementación de sistemas de evaluación del desempeño para la administración pública, esto derivado de que una administración puede contar con planes, proyectos que pudieran trascender en el tiempo, pero que resultan ser interrumpidas debido a la llegada de nuevas administraciones y no les interesa preservar lo que otro inició. 


\section{Conclusiones}

Corresponde a los servidores públicos respetar y hacer cumplir la normatividad vigente, garantizar el bien común, así como procurar el bienestar de los ciudadanos. El problema surge cuando un servidor falta o se conduce de manera incorrecta anteponiendo los intereses propios a los de la sociedad en busca de algún beneficio. Dichos actos deben ser sancionados por la gravedad de las faltas, el daño causado y la jerarquía de quien los comete. De lo anterior descrito, resulta la importancia de contar con un código de ética en nuestros lugares de trabajo que contengan los principios y valores a considerar en el desarrollo de nuestro trabajo con la finalidad de guiarnos en el marco de nuestro actuar.

Alcanzar un nivel de excelencia en el actuar de los servidores públicos dentro de la administración pública, es una tarea difícil, y no será posible a menos que se contemple la formación ética como un mecanismo para reforzar las conductas; así mismo, es necesario el establecimiento de un perfil público deseable del servidor al momento de llevar a cabo contrataciones a fin de evitar el ingreso a la administración pública de personas que no cumplan con dicho perfil y por consiguiente pudieran adoptar conductas indebidas, fomentando con malas prácticas y con ello la corrupción que es la causa principal por la desconfianza que existe en el gobierno y sus instituciones.

La Ética pues, es un factor clave para elevar la calidad de la administración pública mediante una conducta honesta e integra de sus funcionarios.

\section{Referencias}

Aldeguer, B. (2011). La Ética Pública en la Configuración del Nuevo Servicio Público. Revista Agenda Pública , 13.
Alonso Súarez, L. (2014). La dimensión ética de la Función Pública. Revista de Investigaciones Póliticas y Sociológicas , 241.

Ausín Díez, T. (2015). Ética Pública para generar Confianza. Revista Vasca de Gestíon de personas y organizaciones públicas , 37.

Boehm, F. (2015). Códigos de Comportamiento para la Administración pública. Revista Digital de Derecho Administrativo , 72.

Casas Aranda, R. (2015). Ética de la Función Pública. Especial referencia al ambito local. Aletheia: Cuadernos Críticos del Derecho , 124-127.

Cuevas Moreno, R., \& Rodríguez Minor, R. (2017). Responsabilidad social y ética profesional en el gestión de la administración pública y empresarial. Revista Científica Pensamiento y Gestón , 7.

Diego Bautista, O. (2007). Ética en la Gestón Pública: Fundamentos, Estados de la cuestión y procesos para la implementación de un sistema ético integral en los gobiernos. Madrid, España: Universidad Complutense de Madrid.

Diego Bautista, O. (2005). La Ética y la corrupción en la Política y en la administración Pública. Mexico, D.F.: Universidad Autónoma del Estado de México.

Jimenez Asensio, R. (2013). Ética Pública, Política y Alta Administración. Los códigos éticos como via para reforzar el buen gobierno, la calidad democrática y la confianza de la ciudadania en sus instituciones. Revista Vasca de Gestíon de personas y organizaciones públicas , 56 .

Mariñez Navarro, F. (2011). Reflexiones sobre las Éticas en la Gestion Pública. Revista Administración \& Desarrollo , 85. 
Mota Diaz, L. (2005). Promover la Ética: una necesidad Urgente para el Desarrollo Humano y Sostenible en América Latina. Convergencia: Revista de Ciencias Sociales , 420.

Naessens, H. (2010). ÉTICA PÚBLICA Y TRANSPARENCIA. Toluca: Universidad Autónoma del Estado de México.

Rafael, C. A. (2015). Ética de la Función Pública. Especial referencia al ambito local. Aletheia: Cuadernos Críticos del Derecho , 123-127.

Rodríguez-Arana Muñoz, J. (1997). Ética y Empleados Públicos. Jornadas sobre el estudio del Estatuto de la Función Pública (pág. 29). Madrid: Fundación Fernando de los Ríos.

Sanromán, R. (2014). Capitulo 1: Economía Legal, Ética, Valores y la Transparencia en la Administración Pública. Santa Elena, Ecuador: Ecorfan.

Seijo Suárez, C., \& Añez Tellería, N. (2008). La Gestión Ética en la Administración Pública: Base Fundamental de la Gerencia Ética de Desarrollo. Revista del Centro de Investigación de Ciencias Administrativas y Gerenciales, 18.

Uvalle Berrones, R. (2014). La importancia de la ética en la formación del valor público. Revista Estudios Póliticos , 60.
Villoria Mendieta, M. (2015). Ética en la administraciones públicas: de los principios al marco institucional. Revista Vasca de Gestíon de personas y organizaciones públicas , 9 .

\section{Leyes y Reglamentos}

Comisión de Ética Profesional del IMCP. (2015). Código de Ética Profesional. Mexico, DF: Instituto Mexicano de Contadores Públicos.

Congreso del Estado Libre y Soberano de Sonora. (18 de Julio de 2017). Ley 191. Ley Estatal de Responsabilidades . Hermosillo, Sonora, Mexico: Boletín Oficial del Gobierno del Estdo de Sonora.

Secretaría de la Contraloría General. (2014). Código de Ética y Conducta de los Servidores Públicos del Poder Ejecutivo del Estado de Sonora. Hermosillo Sonora: Gobierno del Estado de Sonora.

\section{Sitio Web}

Secretaría de la Función Pública. (9 de Septiembre de 2013). gob.mx. Recuperado el 26 de Mayo de 2018, de Tu gobierno en un solo punto: https://www.gob.mx/sfp/documentos/definicion-decorrupcion 